\title{
Intracranial Extension of Fungal Sinusitis
}

\author{
${ }^{1}$ Nishit J Shah, ${ }^{2}$ Anamika Rathore \\ ${ }^{1}$ Honorary ENT Surgeon, Bombay Hospital and Medical Research Center, Mumbai, Maharashtra, India \\ ${ }^{2}$ ENT Surgeon, The Cumballa Hill Hospital and Heart Institute, Mumbai, Maharashtra, India
}

Correspondence: Nishit J Shah, Honorary ENT Surgeon, Bombay Hospital and Medical Research Center, Mumbai, India House No. 2, Second Floor, Kemp's Corner, Mumbai-400036, India, Phone: +91-22-23802875

\begin{abstract}
Intracranial extension is the most dreaded complication of fungal sinusitis with high mortality rates. Fungal sinusitis is notorious for insidious and rapid intracranial spread. Therefore the most appropriate approach lies in timely diagnosis and early intervention.

With various diagnostic modalities available; like CT scan, MRI, fungal stains, cultures and nasal endoscopy, the diagnosis has become much simpler, nevertheless, a high degree of clinical suspicion is a must.

The treatment outcomes have now improved a lot, with the advancement in medical and surgical technology. The new antifungal agents and other drugs have immensely contributed to better results by minimizing the toxic side effects of the traditional drugs and showing better efficacy. The surgical approach, with the advent of endoscopes has led to successful outcomes.

Despite this, in about $50 \%$ of the cases, since the host is immunocompromised, the prognosis is guarded and mortality rates of approximately 85 to $100 \%$ are noted.

So, it is very important to treat intracranial extension of fungal sinusitis as a medical and surgical emergency.

Keywords: Intracranial extension, fungal sinusitis.
\end{abstract}

\section{INTRODUCTION}

The term fungal sinusitis has a sinister reputation due to infrequent but fatal intracranial extension, irrespective of the etiology, whether invasive or noninvasive. Usually, it's a consequence of the former. It has to be treated urgently and aggressively as it invariably proves to be fatal. Most of the time, a delay in diagnosis can lead to a disastrous outcome. Therefore, clinical suspicion must be present in cases of refractory sinusitis or long-term sinus disease.

The extension is either direct into the skull base due to proximity of the sinuses, or due to pressure necrosis in case of long standing infections. It could be blood borne in some cases.

The presentation could be symptomatic or asymptomatic. In case of doubt, MRI can be done to assess the intracranial spread, especially when erosion of the sinus walls is seen on the CT scan. Since the mortality rates are very high with the intracranial disease, an early diagnosis and treatment is the best option. With the invention of newer and more effective, less toxic drugs, the medical treatment has reduced the mortality to some extent. This, combined with surgery has improved the results marginally.

The intracranial extension generally presents with neurological symptoms, and the patient often consults a neurosurgeon or an ophthalmic surgeon. So, it is very important for these surgeons to recognize fungal sinusitis as the cause. Although, it seems that this entity is more common in the immunocompromised, especially poorly controlled diabetic patient, it is also prevalent in the immunocompetent population.

The prognosis is dependent on the invasiveness of the fungus, and is the best for allergic fungal sinusitis, leading to intracranial extension in immunocompetent patients. It is worst in case of mycotic aneurysms caused by aspergillus or mucormycosis, where mortality ranges from 85 to $100 \%$.

Due to hot and dry tropical climate in India and South East countries, aspergillus spores are prevalent in the environment leading to fungal infections. This compounded by a myriad of predisposing factors like poor nutrition, low immunity, diabetes, antituberculous treatment, antibiotics, etc people in these countries are more prone to these infections.

\section{ETIOLOGY}

Fungal sinusitis is the inflammation of the sinus mucosa, caused by a wide variety of fungi. Aspergillus is the most common and rhizopus, mucor, cladosporium, candida, cryptococcus are amongst the others. The noninvasive ones 
are generally, demateacious molds including curvularia, bipolaris, alternaria, fusarium, aspergillus, etc. These cause intracranial complications in about $20 \%$ of the patients.

There are broadly two types of fungal infection of the sinuses $^{3}$

1. Noninvasive (extramucosal)

- Allergic fungal sinusitis

- Mycetoma.

2. Invasive

- Acute

- Chronic indolent

- Chronic granulomatous.

Noninvasive: These generally don't invade the bone or tissues and more often, are a result of hypersensitivity skin reactions. But a long-term disease can eventually erode the bone by pressure necrosis and hence cause an intracranial or intraorbital complication. It occurs in an immunocompetent host and is characterized by presence of allergic mucin, charcot leyden crystals, eosinophils, etc.

Invasive: The more fatal variety, known to penetrate the mucosa and cause tissue destruction and lead to intracranial extension. These are known to occur in immunocompromised hosts in about $50 \%$ of the cases. The most common is aspergillus followed by mucor, rhizo, cryptococcus, etc. The mortality rates are high in such infections (85-100\%).

The acute variety rapidly progresses in few hours to days and progresses to fulminant intracranial infections. Mostly, these are caused by mucor, absidia, fusarium, etc. Some authors prefer to call such infections, zygomycosis or rhinocerebral mucormycosis.

These often occur in patients with diabetes having ketoacidosis.

The chronic one has an indolent course and could be granulomatous also. The species implicated are alternaria curvularia, mucor, bipolaris, etc. These are slow growing and cause slow tissue destruction and subsequent invasion.

\section{MODE OF SPREAD}

The intracranial extension is one of the most dreaded complications of fungal sinusitis with high mortality rates.

The fungus generally spreads through:

1. Direct extension.

2. Hematogenous route.

3. Perineural invasion of cranial nerves.

4. Cribriform plate of ethmoid bone.

5. Rarely - surgery or blood transfusion.
Direct extension: This is the most common mode of extension. Due to the central location of the sphenoid sinus, the infection can easily spread to the surrounding skull base structures like cavernous sinus, sella, internal carotid artery, meninges, clivus, prepontine cistern, rarely basilar artery, etc.

The fungus causes osteitis and osteomyelitis of the sinus wall/pressure necrosis, and subsequent erosion leading to the extension in the anterior skull base. ${ }^{10}$ A direct vascular invasion is caused by extension of fungal hyphae in the CNS leading to aneurysmal dilatation. The invasion causes an acute inflammatory reaction resulting in secondary cerebral infarction. As the fungus is known to be angioinvasive; it digests the elastic tissue and penetrates the vessel wall, causing arteritis and intramural spread. In direct arterial involvement, the endothelial cells engulf the organism and subsequently the internal elastic lamina is infiltrated and destroyed. The fungus has a predilection for the arteries with multiple perforators like basilar, thus causing subarachnoid hemorrhage.

In acute cases the fungal organisms invade the mucosa, submucosa, blood vessels, and bony walls of the sinuses and nasal cavity. ${ }^{7}$

Hematogenous: It is more insidious and asymptomatic. There is also formation of mycotic emboli and thrombus due to extension of fungal hyphae. The fungus usually involves more proximal portions of the vessels causing vascular damage in large areas. These are more common in immunocompromised patients. The thrombus or the emboli leads to cerebral infarction. The primary could be in the lungs, as in case of aspergillus sp. It is also likely in patients with artificial valves in heart. This spread could lead to vasculitis and eventually meningitis or cavernous sinus thrombosis.

Perineural spread: It occurs along the nerves and their foraminae resulting in cranial nerve palsies and anterior skull base spread.

The infection can also spread through the cribriform plate, into the anterior skull base. Rarely, a blood transfusion can cause such infection. It has also been reported to be spread by surgery, especially trans-sphenoidal.

\section{EXTENSION}

The various mechanisms of the intracranial spread have been elicited above. The various intracranial complications are listed below: 
- Cavernous sinus thrombosis

- Meningitis

- Intracranial fungal masses (IFM)/granuloma

- Brain abscess

- Cranial nerve palsies

- Cerebritis

- Stroke

- Mycotic aneurysm.

Cavernous sinus thrombosis: It is a potentially fatal complication and may require an intervention in collaboration with a neurosurgeon. It is usually a consequence of a hematogenous spread. Due to its close proximity to the sphenoid sinus, it tends to get involved more commonly. The usual presenting symptoms are: Ophthalmoplegia, chemosis, visual loss, and involvement of the cranial nerves like abducens, V2/V3. 3 or 4 cranial nerves (Figs 1 and 2).

Meningitis/meningoencephalitis: It is a consequence of hematogenous spread, causing vasculitis, and inflammation of the meninges. The patient presents with meningismus, neck pain, rigidity, fever. The primary focus is generally the paranasal sinuses and a careful history and high degree of clinical suspicion is required to diagnose the fungus.

Brain abscess: This could be intraparenchymal/epidural. It could be silent at times, or present with altered sensorium, persistent fever, and severe headaches. This could be a result of direct spread from the paranasal sinuses, with focal bony erosion.

Intracranial fungal masses: These are not so common and generally present as space occupying lesions. More often, the spread tends to be hematogenous, but direct extension from paranasal sinuses and mastoid is also present. These masses can occur in age groups as young as neonates/infants to older age groups like 5, 6 decades. They occur in immunocompetent patients in 50\% cases, generally caused by Aspergillus flavus. The immunosuppressed patients are infected by A. fumigatus, though several other species like mucor, cryptococcus, candida, etc have been implicated.

The predisposing factors could be diabetes mellitus, renal transplant, long-term immunosuppressant with steroids, parasitic infections, rampant use of antibiotics, antituberculous therapy, poor nutritional status, leading to reduced host defense, longer survival rates, prevalence of HIV infection and rarely surgery. The tropical hot and dry climate promotes aspergillus spores and contributes to its wide spread prevalence in our country. ${ }^{5,9}$

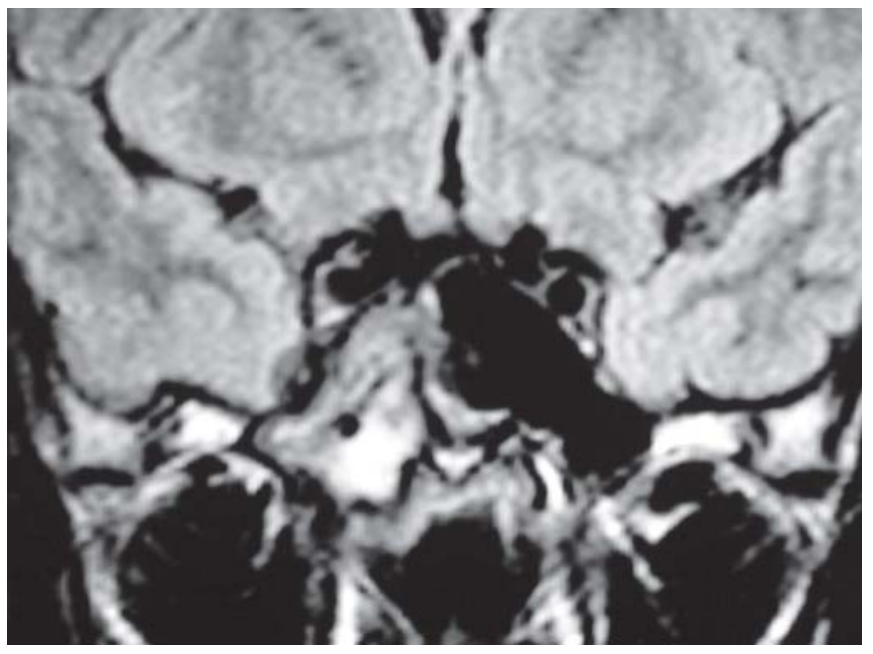

FIGURE 1: Invasive fungal sinusitis—involving right cavernous sinus and ICA-MR

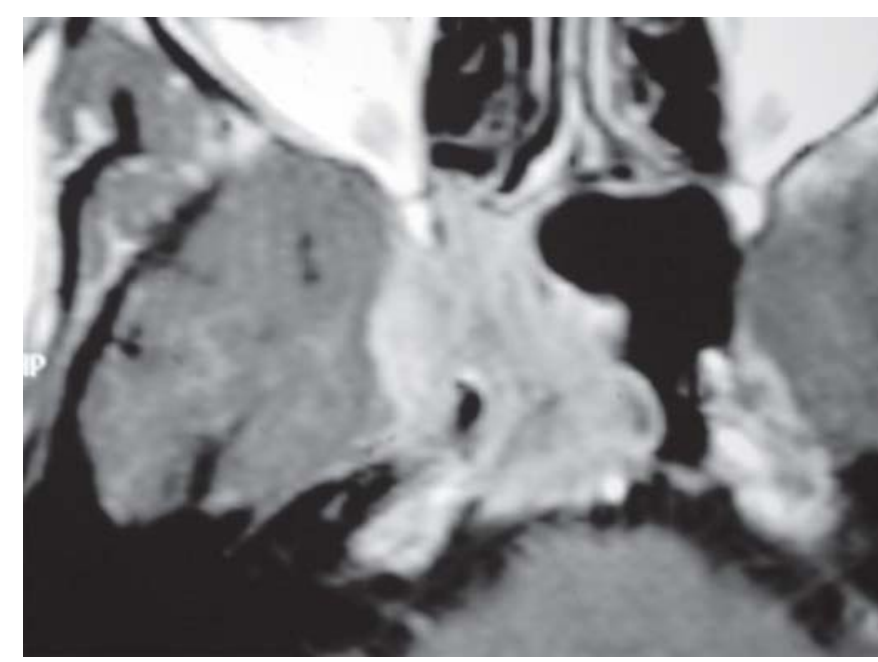

FIGURE 2: Invasive fungal sinusitis—involving right cavernous sinus and ICA-MR axial

The clinical symptoms could be involvement of cranial nerves 1 to 6 with orbital and nasal symptoms, focal neurological deficits, or stroke like symptoms. They are divided into rhino cerebral or pure intracranial masses. The former spreads directly from the infected paranasal sinuses, either from bone erosion in case of sphenoid sinus or extension through the cribriform plate (Figs 3 and 4).

There presenting symptoms include headaches, nasal obstruction/discharge, anosmia, weakness of limbs, diplopia, facial pain/heaviness, altered sensorium, fever, refractory sinusitis, etc.

The signs could be those of raised intracranial pressure, proptosis, visual blurring, cranial nerve palsy, hemiparesis, etc. 


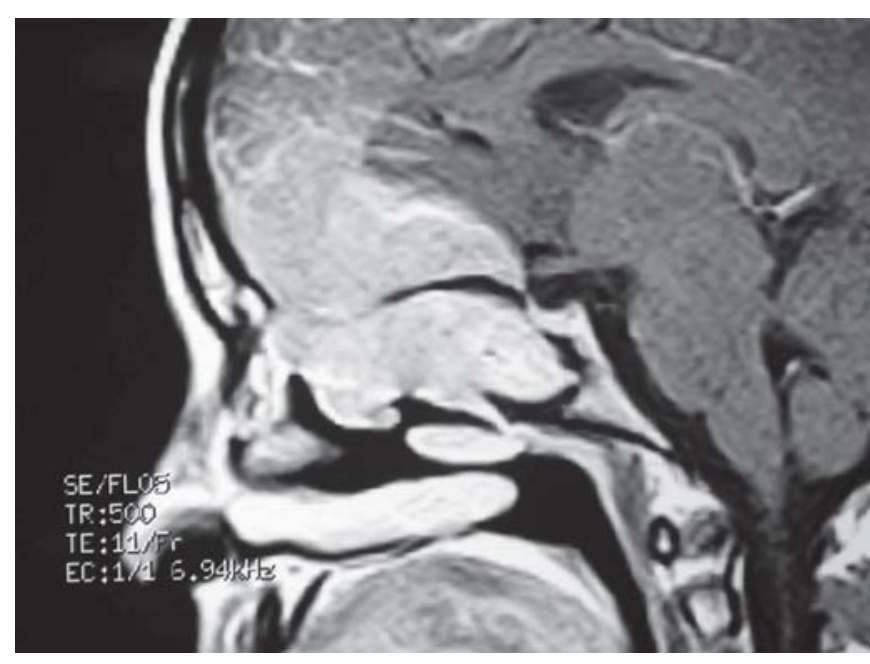

FIGURE 3: Invasive fungal sinusitis-anterior cranial fossa extension -MR parasaggital

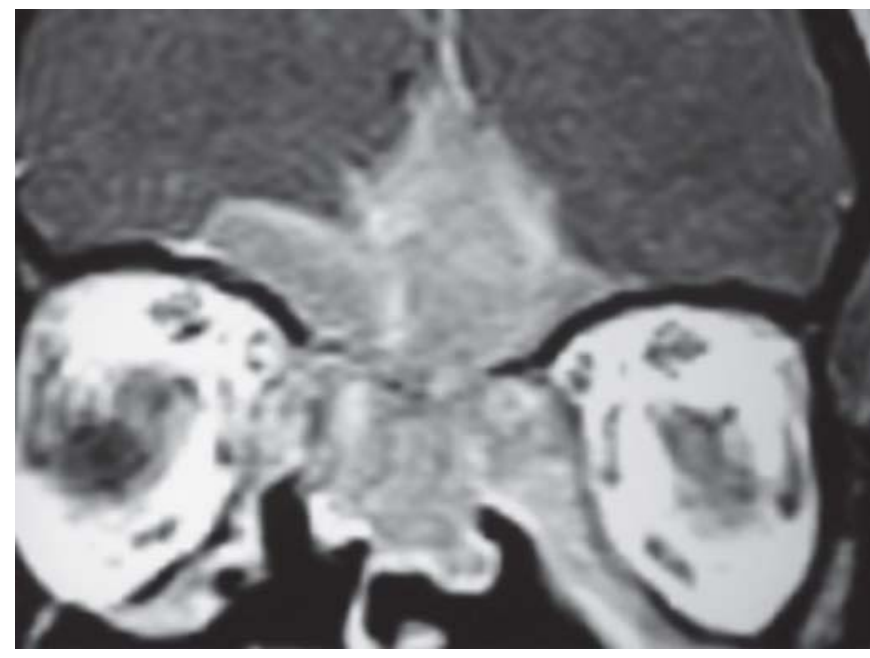

FIGURE 4: Invasive fungal sinusitis—anterior cranial fossa extension -MR coronal

Mycotic aneurysms: These are rare entities and only few cases have been reported so far. They are almost invariably fatal with mortality rates of 85 to $100 \%$. They are mostly caused by aspergillus. They are known to invade the blood vessels as described above and cause aneurysmal dilatations leading to cerebral infarction/subarachnoid hemorrhage and death. They grow intraluminally in blood vessels and have been reported in internal carotid artery and basilar artery with fatal outcome.

They can present with epistaxis, subarachnoid hemorrhage, fusiform dilatation of the vessels and have guarded prognosis. ${ }^{1}$

Cranial nerve palsies: The fungus can either travel through the skull base foramina directly or through hematogenous route.
There is 3,4,6 nerve palsy noted. Sometimes isolated third nerve palsy is also seen. The patient can also present with V1,2,3 palsy.

\section{COMPLICATIONS}

These are generally secondary to the intracranial extension. These are usually cerebral infarcts or subarachnoid hemorrhage. There is occasional ischemia and could lead to stroke. These complications are almost invariably fatal and should be treated urgently.

\section{DIAGNOSIS}

A good history along with symptoms of any systemic ailments should be elicited. A sinusitis patient not responding to conventional antibiotics should arouse suspicion.

Diagnostic nasal endoscopy should be performed and one should look for allergic mucin, blackish/brownish discharge, erosion of palate, pale/dark nasal mucosa, etc.

\section{SYMPTOMS/SIGNS}

The most common symptoms are nasal obstruction, nasal discharge, headache, vomiting, nausea, epistaxis, periorbital pain, facial pain, facial swelling, anosmia, altered sensorium, seizures, and weakness of limbs, diplopia, visual disturbance and fever.

The signs depend upon the areas involved like ophthalmoplegia, chemosis, proptosis, lateral/medial rectus palsy, hemi paresis.

\section{INVESTIGATION}

Fungal culture and biopsy: There are special stains required for detecting the fungus. These include methamine silver for mucor, rhizopus, absidia. The aspergillus has uniform septate hyphae branching at 45 degree. The mucor shows nonseptate, nonuniform branching at 90 degrees. The allergic mucin is characteristic of AFS and one has to stain deeply to look for fungal hyphae. ${ }^{8}$

The culture used is sabauraud media.

The biopsy especially from the middle turbinate is helpful in diagnosing the fungus. All the material including soft tissue and bones should be sent for histopathological examination.

\section{RADIOLOGY}

Since fungal infections are rapidly on the rise resulting in disastrous outcomes, one has to diagnose them early with 
the aid of radiology. The CT scan and MRI of the PNS and brain are the mainstay in diagnosing these infections.

There is generally a homogenous mass with areas of lesser density, linear interlacing pattern, or dense in center with low attenuation at periphery. There could be expansion, thinning, erosion or remodeling of the bone with intracranial /intraorbital extension. On contrast enhancement is seen. For intracranial extent an axial cut is also needed. ${ }^{4}$

CT scan: Noncontrast-The early stages resemble chronic rhino sinusitis showing mucosal thickening which is hypoattenuated. Since the fungus may spread by blood vessels, bone erosion or mucosal thickening might be absent.

In chronic cases, a hyper attenuating soft tissue collection can be seen in one or more sinuses. It often gives a mass like appearance, resembling a malignancy with destruction and erosion of the bony walls of the sinuses and extension to the surrounding tissues.

In case of allergic fungal sinusitis, there is opacification of multiple sinuses with expansion. There are areas of hyper attenuation in the sinuses due to the presence of allergic mucin. It is essential to have both bone window and soft tissue windows to appreciate the 'double density' (Figs 5 and 6).

When a fungal ball is present it appears as hyper attenuation due to dense matted fungal hyphae and may show punctate calcification.

There is mottling seen in some cases with sclerotic walls of the sinus, along with thinning due to pressure necrosis.

MRI: Since a CT scan only reveals a bony destruction, an MRI is needed for assessing the intracranial and intraorbital spread.

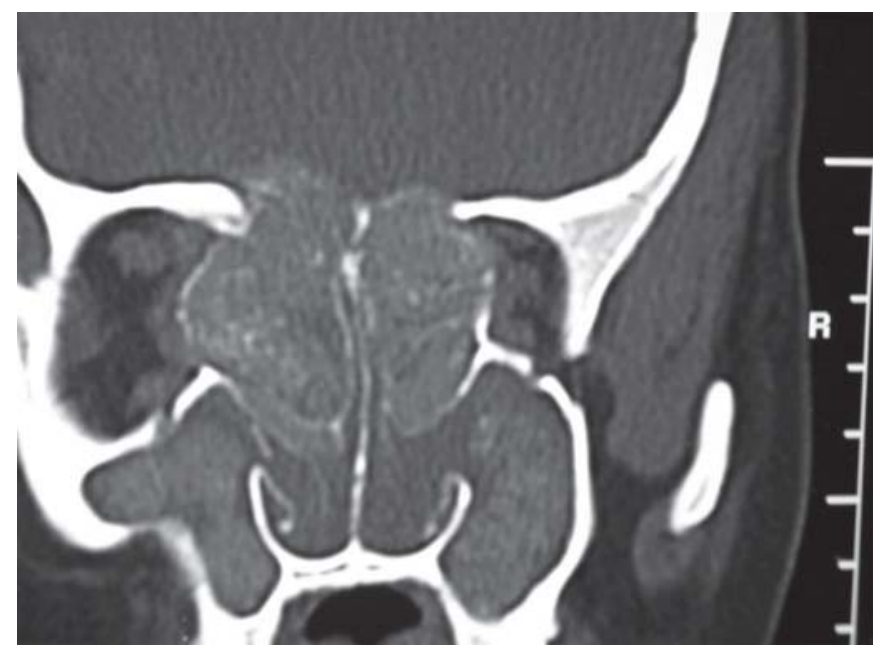

FIGURE 5: Early intracranial extension of AFS - bone window coronal CT scan

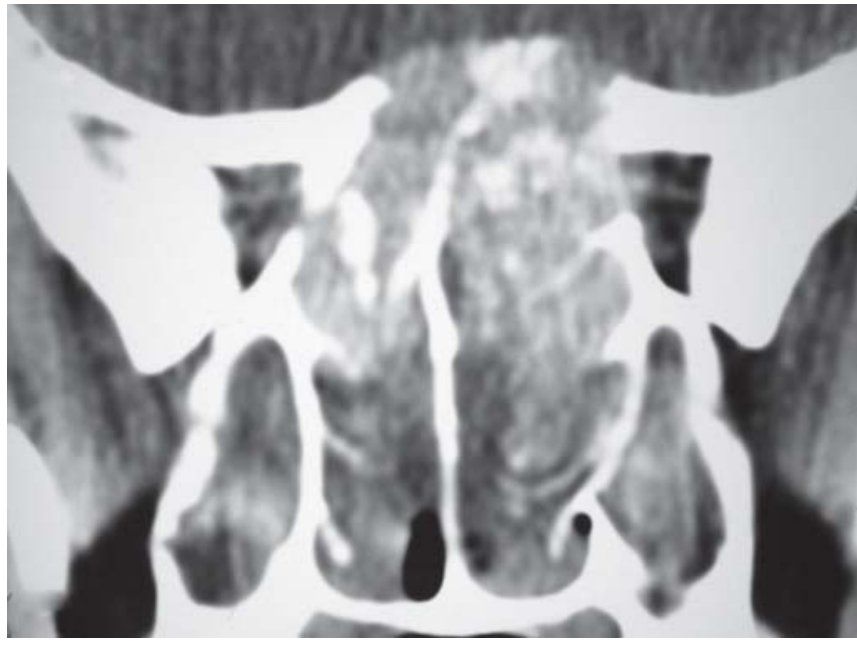

FIGURE 6: Early intracranial extension of AFS - soft tissue window coronal CT scan

In acute cases, one should look for obliteration of periantral fat planes. Sometimes, leptomeningeal enhancement is seen with intracranial spread. The granulomas might appear as hypointense masses on T1-T2-weighted images with very little enhancement on contrast.

In diagnosing chronic invasive cases, infiltration of periantral soft tissue around the maxillary sinus should be looked for. The T2-weighted images have markedly low intensity.

In case of AFS there is hyperintensity on T1 and hypointensity on T2 of the sinus contents. The inflamed mucosa shows hyperintensity on $\mathrm{T} 2$ and enhancement on contrast. The center is devoid of the enhancement, unlike a malignancy (Figs 7 to 9).

A fungal ball is hypointense on both T1 and T2 and shows signal void on T2 due to calcification and paramagnetic metals like iron, magnesium and manganese. ${ }^{2}$

\section{MANAGEMENT}

\section{Medical}

The mainstay of treatment is antifungal therapy with amphotericin B. It combines with the ergosterol in fungal cell membrane and manifests its antifungal properties. The maximum dose is $2-4 \mathrm{gm} /$ day. Due to its toxic effects, the Liposomal ampho B is preferable, as it is less toxic. Due to its high cost, it is generally used in patients having creatinine $>2.5 \mathrm{mg}$. The required dosage is $4 \mathrm{mg} / \mathrm{kg} /$ day and could be increased up to $10-15 \mathrm{mg} / \mathrm{kg} / \mathrm{day}{ }^{6}$

We can also use azoles, as they act by blocking ergosterol. It has been shown that Voriconazole is very 


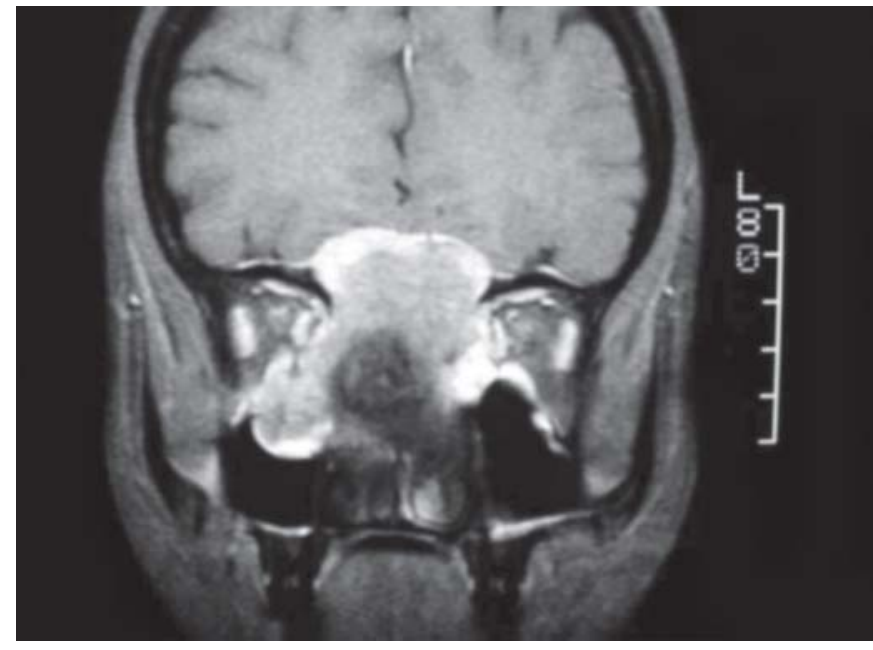

FIGURE 7: Extradural intracranial extension of AFS with significant bone erosion-MRI

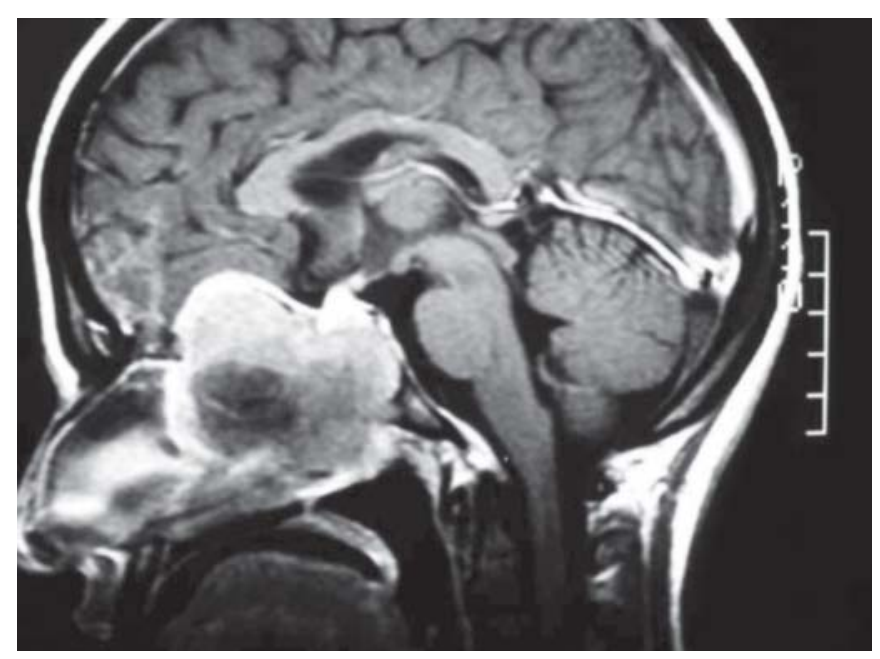

FIGURE 8: Significant extradural intracranial extension of AFS-MRI parasagittal

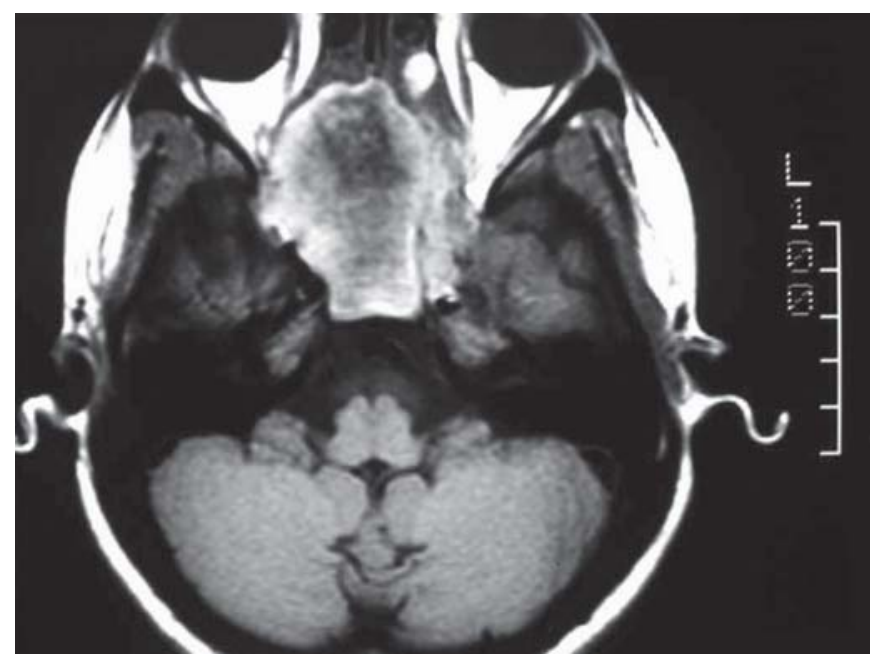

FIGURE 9: Significant extradural intracranial extension of AFS-MRI axial effective in complicated cases and is less toxic than amphotericin. Apart from this fluconazole or itraconazole $400 \mathrm{mg}$ twice daily, may be used.

The studies have shown (The New England Journal of Medicine), that a new molecule echinocandin to be useful as an antifungal. The prototype is Capsofungin, which acts on the cell membrane.

The recombinant interferon gamma - IFN can also be used, as it improves antifungal properties of polymorphs and helper T cells.

GMCSF 200 microgram thrice weekly can be used.

Amphotericin can also be used topically or instilled in the cavity.

The complications like cavernous sinus thrombosis and meningitis respond very well to intravenous antibiotics like cephalosporins and amikacin, in additional to the above treatment.

\section{Surgical}

An initial biopsy and culture (endoscopic directed) is taken to confirm the presence and type of fungal involvement. Intracranial stereotactic biopsy can be taken, if sinus tissue is absent.

In cases of obvious clinical fungal sinusitis, endoscopic sinus surgery with removal of all fungal debris should be performed even at the initial stage when sending material for histopathology and microbiologic studies.

There are various surgical options in established fungal sinusitis cases with intracranial extension.

If the disease is not invasive and the fungus remains extradural, like for example in allergic fungal sinusitis, then the obvious choice is endoscopic clearance. Occasionally large areas of bone erosion are present, but despite this, it is usually possible to remove all allergic fungus without having a CSF leak. The aim is to have a large exposure and avoid using sharp instruments. We have found the blunt curettes, suction and irrigation are safe and effective in removing such fungus in proximity to the dura. Usually the dura is strong and requires no additional reinforcement. One must ensure the cavity is well marsupialized and stays open postoperative for drainage and inspection. Postoperative image for AFS in figures 7,8 and 9 (Fig. 10).

In cases of invasive fungal sinusitis, one may have to consider nonendoscopic approaches. If the fungus is confined to the sinuses without obvious dural involvement or osteomyelitis, one could then still consider a radical endoscopic approach. Unlike FESS, mucosa is stripped off 


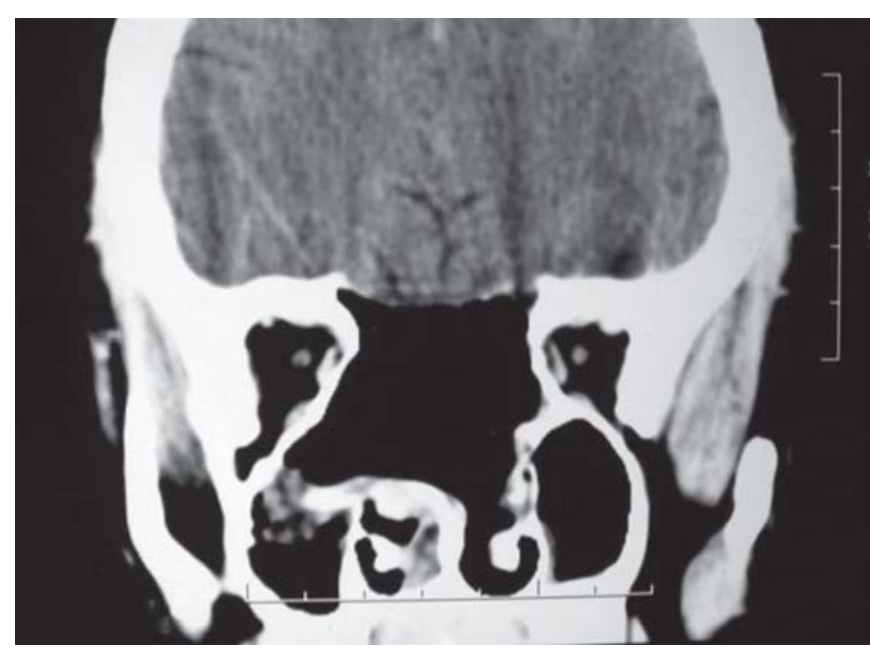

FIGURE 10: Postoperative scan of AFS showing clear cavity and dura of planum sphenoidale

with the disease (except in uninvolved areas), wide meatotomies are made and turbinectomies are performed even on suspicion of fungus involvement. When there is osteomyelitis, and extensive bone removal is required, then transfacial procedures will be required. If there is dural involvement, then additional transcranial surgery is done in a team approach with the neurosurgeons. These surgeries may include craniofacial exposure and resection to include the sinonasal and intracranial areas. Bifrontal osteoplastic flap surgery will help in anterior cranial fossa spread while exposing the frontal sinus as well. In case of severe cases craniotomy or ventriculoperitoneal shunt is additionally required.

Other transcranial approaches may be used to access the middle or posterior cranial fossa as required by the neurosurgical colleagues. There will certainly be instances when the fungus is not easily approachable without significant morbidity and mortality (as in cavernous sinus spread or mycotic aneurysms), and in these situations, one may have to depend on medical treatment alone. For small residues in the cavernous sinus one may consider the option of gamma knife or $x$-knife radiotherapy but we are unaware of any studies discussing the role of radiotherapy for intracranial fungal disease. Areas which can be cleared such as the sinuses or the middle compartments must however be operated so as to reduce the fungal load. The principles of treatment of intracranial spread of fungal sinusitis remain the same, namely - early detection, aggressive surgery, control of underlying systemic problems (such as diabetes, immunosuppression, etc) and antifungal medical therapy.

\section{POSTOPERATIVE CARE}

A regular follow-up and endoscopic examination is essential. The patient is asked to do nasal wash twice a day. Dilute Amphotericin-B washes may be used as well. The antifungal agents are continued for about 4 weeks or till the endoscopic examination reveals fungus free healed mucosa.

Postoperative neurologic follow-up and assessment is critical. Often serial scans are required to confirm presence or spread of disease postoperatively.

\section{CONCLUSION}

The intracranial extension of fungal sinusitis should be treated as a medical emergency, as the outcome is fatal in most of the cases. For early diagnosis, a high degree of clinical suspicion is required. The treatment is essentially a combination of medical and surgical procedure, in collaboration with a neurosurgeon. With the evolution of endoscopy and emergence of newer and better drugs, the treatment aspects have improved, leading to less morbidity and mortality. However, one has to be extremely cautious and careful while dealing with such cases, as the prognosis remains guarded, despite the progress in medical technology.

\section{REFERENCES}

1. Ahsan H, Ajmal F, et al. Cerebral fungal infection with mycotic aneurysm of basilar artery and subarachnoid haemorrhage. Singapore Medical J 2009;50(1):e22.

2. Arihandi M, McCoy V A, Bazan C 111, Imaging features of invasive and noninvasive fungal sinusitis: A review, Radiographics 2007;27:1283-96.

3. Ballenger's. Otorhinolaryngology Head and Neck Surgery. Part II/Rhinology 504-06.

4. Dahniya MH, Makkar R, et al. Appearance of paranasal fungal sinusitis in CT, The British Journal of Radiology 1998;71:34044.

5. Dubey A, Patwardhan RV, et al. Intracranial fungal granuloma: Analysis of 40 patients and review of literature. Surgical Neurology 2005;63:254-64.

6. Ellis M, Watson R, et al. Massive intracerebral aspergillosis responding to combination high dose liposome amphotericin $\mathrm{B}$ and cytokine therapy without surgery. J Med. Microbiology 2002;51:70-75.

7. Pilch Ben Z. Head and Neck Surgical Pathology, Lippincott Williams and Wilkins 2000;92.

8. Helal. Endoscopic nasal and Skull base Surgery. Chapter 10 Endoscopic Surgery for Fungal Sinusitis.

9. Ravishekhar V. Surgical management of intracranial fungal masses. Neurology India 2007;55(3):267-73.

10. Swift AC, Denning DW. Skull Base Osteitis following Fungal Sinusitis. JLO Jan 1998;112(1):92-97. 